\title{
Metastatic Primary Pulmonary Angiosarcoma
}

\author{
Brendan F. Judy ${ }^{1}$, Jarrod D. Predina ${ }^{1}$, Jay Mittal ${ }^{1}$, Charuhas Deshpande ${ }^{2}$, Sunil Singhal ${ }^{1}$ \\ ${ }^{1}$ Division of Thoracic Surgery, Department of Surgery, \\ University of Pennsylvania School of Medicine, Philadelphia, USA \\ ${ }^{2}$ Department of Pathology and Laboratory Medicine, \\ University of Pennsylvania School of Medicine, Philadelphia, USA \\ E-mail: brendan.judy@gmail.com,judyb@uphs.upenn.edu
}

Received January 18, 2011; revised March 20, 2011; accepted April 2, 2011

\begin{abstract}
Angiosarcoma is an extremely aggressive tumor with a high rate of mortality. It can arise in any tissue of the body and is most commonly found in the skin and soft tissue [1]. Pulmonary angiosarcoma is usually secondary to a primary location of the body and presents as both solitary and multiple nodules. Primary pulmonary angiosarcoma is a rare disease with less than 20 cases reported in the English literature. In our report we present a case of metastatic primary pulmonary angiosarcoma involving the most $(>100)$ pulmonary nodules known to date. Novel treatment strategy using an anti-angiogenic inhibitor was used to treat this disease for the first time to our knowledge; however, it was unsuccessful in controlling disease progression. This report reviews the literature of this rare and devastating disease.
\end{abstract}

Keywords: Angiosarcoma; Pulmonary; Tumor; Surgery

\section{Introduction}

Angiosarcoma of the lung is a rare tumor that most often presents as metastatic disease [2,3]. Rarer still is primary pulmonary angiosarcoma, with less than 20 cases reported in the literature. Patients have been treated with steroids, radiotherapy, surgical resection, immunotherapy and chemotherapy [3]. All except three cases fall into a survival range of 1 - 9 months. The two survivors in the literature are a 25-year-old male who received radiotherapy and immunotherapy with recombinant interleukin-2 (rIL-2) [4], and a 65-year-old male who underwent surgery for tumor removal, a complete pneumonectomy, and chemotherapy using ifosfamidedoxorubicin combination [5]. In this report we describe a patient with innumerable primary pulmonary angiosarcoma nodules who presented with dramatic weight loss and hemoptysis.

\section{Case Report}

A 45-year-old-male nurse was referred to our hospital with $40 \mathrm{lb}$ weight loss over four months, anorexia, hemoptysis, and fatigue. The patient had smoked one pack-per-day for 20 years and recently quit. The patient was otherwise healthy and had no preceding medical problems.

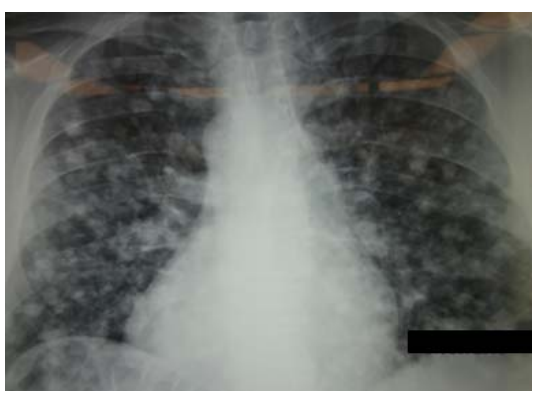

(a)

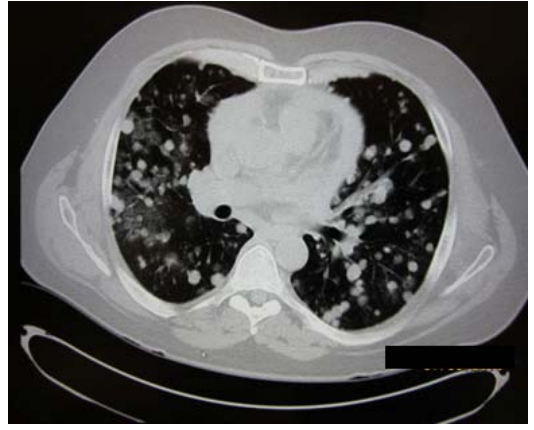

(b)

Figure 1. (a) Posterior - anterior chest $x$-ray demonstrating multiple bilateral nodular opacities and vascular crowding. (b) A contrast enhanced computed tomography examination of the chest revealing over 100 bilateral pulmonary nodules. 
Two years prior to presentation, the patient had a chest $\mathrm{x}$-ray that revealed no abnormalities. On admission, a posterior-anterior chest radiograph demonstrated multiple pulmonary nodular opacities with vascular crowding (Figure 1(a)). A chest computed tomography (CT) scan showed innumerable bilateral nodules $(>100)$ varying in size from a few $\mathrm{mm}$ to $18 \mathrm{~mm}$ with scattered areas of groundglass opacities (Figure 1(b)). A pericardial effusion was noted and measured 35 Hounsfield units. Degenerative changes were seen in the thoracic spine but the great vessels, heart, and airways were all normal in appearance. Imaging of the head, abdomen and pelvis were unremarkable. The patient underwent a muscle sparing limited thoracotomy on the right side with multiple pulmonary wedge resections and a pericardial window for drainage of a bloody pericardial effusion. His postoperative course was uneventful and he was discharged from the hospital on postoperative day four.

Pathological examination revealed multiple nodules of proliferating and pleomorphic spindle cells suggestive of vascular neoplasm (Figure 2). The tumor cells showed intravascular "hobnailing" and occasional intracytoplasmic lumina (Figure 3(a)). The tumor specimen underwent immunohistochemical staining and was positive for vimentin, CD31, CD34 (Figures 3(b) and (c)). The tumor cells were negative for pancytokeratin (Figure 3(d)), AE1/AE3, CK7, CAM 5.2, TTF-1, p63, thyroglobulin, S100, SALL 4, HMB45, calcitonin, synaptophysin, EMA, and HHV-8. The histology and immunohistochemistry of the tumor were consistent with angiosarcoma $[2,3,6]$. The pericardial effusion was negative for malignant cells.

The patient was started on bevacizumab, a vascular endothelial growth factor A inhibitor, and paclitaxel. He received three courses of paclitaxel and underwent repeat imaging, four months after surgery, which revealed progression of his pulmonary disease, liver metastases

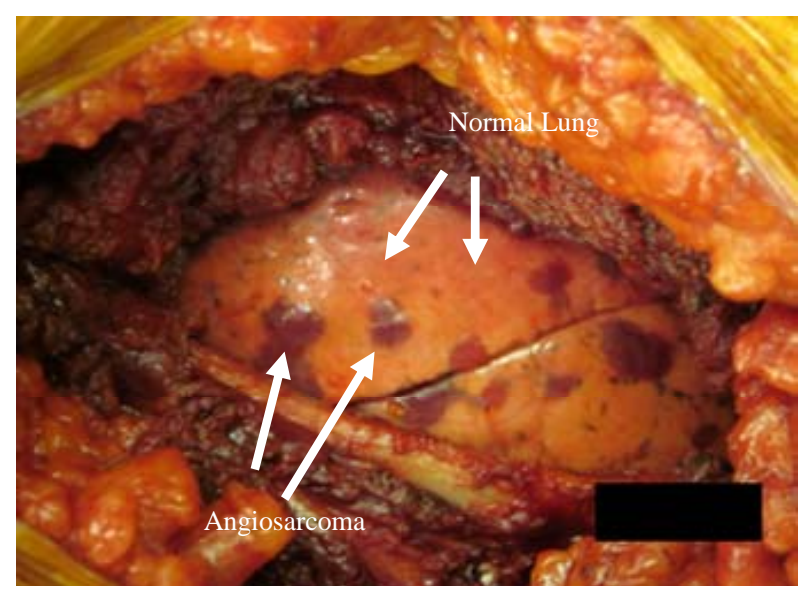

Figure 2. Multiple pulmonary nodules revealed during open surgical biopsy. When palpated, these nodules were soft, non-mobile and non-blanching.

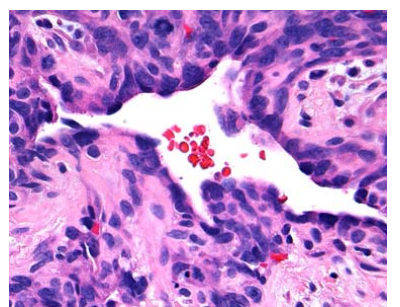

(a)

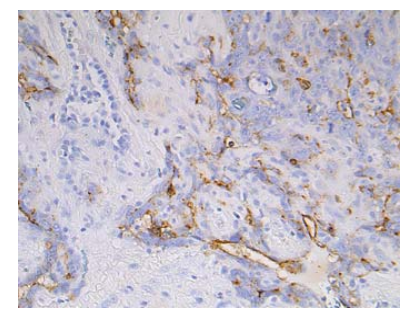

(c)

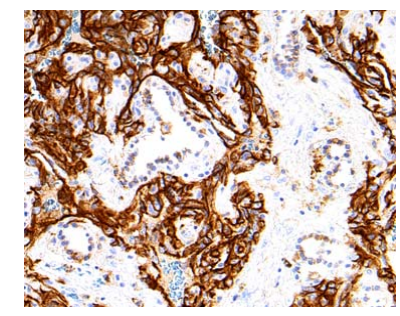

(b)

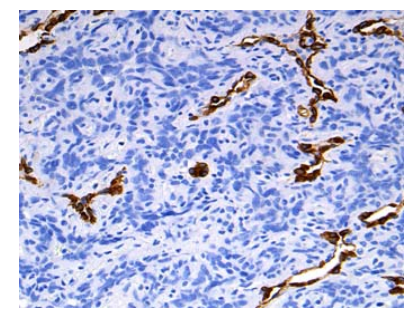

(d)
Figure 3. (a) Hematoxylin and eosin (H\&E) staining demonstrates poor cell differentiation, hobnailing, and intracytoplasmic lumina, 40x. (b) Malignant cells immunoreactive to CD31, 40x. (c) Malignant cells immunoreactive to CD34, 40x. (d) Malignant cells not immunoreactive to pancytokeratin, $40 \mathrm{x}$.

and solitary brain metastasis. Paclitaxel was stopped and dexamethasone was started for two months and tapered off. The patient received whole brain external beam irradiation (3000 rads) and was started on sorafenib, a small molecular inhibitor of several tyrosine protein kinases and Raf. Despite this approach, the pulmonary disease progressed and the patient expired five months following diagnosis from respiratory failure.

\section{Discussion}

Angiosarcoma is a rare tumor that accounts for approximately $1 \%$ of all soft tissue sarcomas [7]. The cause of the pulmonary angiosarcoma is unknown however mastectomy, radiation treatment [8], and exposure to polyvinyl chloride, Thorotrast [2], and copper mining dusts [9] have all been suggested as associated to the disease [7]. Pulmonary sarcomas are most often the result of metastasis from a primary site such as skin, bone, liver, breast, or heart [2-4]. Males are more often affected than females by a 3:1 ratio [10]. There are no symptoms specific to pulmonary angiosarcoma as the presentation is similar to presenting symptoms found in all lung cancers, such as hemoptysis, weakness, dyspnea, and chest pain $[7,11]$. It has been speculated that up to $20 \%$ of all cases are asymptomatic and diagnosis only occurs post-autopsy $[2,10,12]$. Primary pulmonary angiosarcoma presents as both solitary and multiple nodules. Although multiple nodules have been noted before $[1,7,13,14]$, there have been no reports of innumerable $(>100)$ nod- 
ules as we present in our case. A literature search for "primary pulmonary angiosarcoma” yielded 15 articles, which indicates the lack of literature on the topic.

In order to properly diagnose primary pulmonary angiosarcoma it is critical to exclude primary tumors elsewhere in the body [11]. Diagnosis requires a CT scan or magnetic resonance imaging of the body. The CT scan of pulmonary angiosarcoma has a distinctive appearance of soft tissue attenuation surrounded by groundglass attenuation which is also known as the halo sign [7]. The halo is thought to represent the hemorrhage in the surrounding lung due to the nodule [7]. Furthermore, biopsy is necessary in order to confirm the histological and immunohistochemical characteristics of the cancer. Angiosarcoma is positive for endothelial cell markers (CD31, CD34, factor VIII related antigen, and Ulex europaeus agglutin-1 [UEA-1]), epithelial cell markers (cytokeratins, EMA), and vimentin [2,3,12]. Staining of epithelial markers (keratins and EMA) is variable [3] and in our case is negative.

Currently, the therapeutic options for primary pulmonary angiosarcoma include but are not limited to steroids, radiotherapy, surgical resection, immunotherapy and chemotherapy [3]. There is no standard regimen, yet surgery has been the mainstay for locally confined disease [3]. Due to the rarity and aggressive nature of primary pulmonary angiosarcoma, early detection is uncommon and prognosis is poor. The cancer is usually inoperable at the time of diagnosis, as in this case. Only two survival cases have been reported in the literature, yet one survivor was significantly younger (25 years old) than other reported cases $[4,5]$. The 25-year-old survivor received radiotherapy and immunotherapy with recombinant interleukin-2 intratumoral injection. Previous studies have shown that angiosarcoma is responsive to radiotherapy $[15,16]$. Systemic administration of high dose recombinant interleukin-2 (rIL-2) has been shown to inhibit pulmonary metastasis of angiosarcoma of the skin [17]. The mechanism of rIL-2 is thought to involve direct inhibition of tumor cells and activation of natural killer cells and lymphokine-activated killer cells [4, 18]. Two chemotherapeutic combinations have demonstrated partial and full effects: doxorubicin/ifofsamide and docetaxel/gemcitabine. Doxorubicin and ifofsamide were used in one of the reported cases of primary pulmonary angiosarcoma survival [5]. Docetaxel and gemcitabine have proved effective in a variety of other malignancies such as esophageal cancer, non-small cell lung cancer, and breast cancer $[3,19,20]$. This combination continues to be explored in a number of clinical trials. The synergism is purportedly due to docetaxel's effect on metabolism and repair, which results in increased integration of gemcitabine into the DNA [3,21,22].

\section{Conclusions}

Primary pulmonary angiosarcoma is a devastating disease with almost a $100 \%$ mortality rate. The diagnosis of this tumor and histological findings were presented. The gold standard for diagnosing this disease is full body imaging in order to rule out other primary tumors and immunohistochemical confirmation. Attempts at novel therapies such as targeted biological agents such as in this patient were unsuccessful. Intuition would suggest that an anti-vascular inhibitor such as bevacizumab would permit some disease regression; however, this was not seen. In addition, a tyrosine kinase inhibitor was also unsuccessful in stabilizing disease progression. Future efforts at biological agents are reasonable, but alternative strategies should be considered.

\section{References}

[1] Y. B. Chen, L. C. Guo, L. Yang, et al., "Angiosarcoma of the Lung: 2 Cases Report and Literature Reviewed,” Lung Cancer, Vol. 70, No. 3, 2010, pp. 352-356. doi:10.1016/j.lungcan.2010.09.002

[2] A. M. Patel and J. H. Ryu, "Angiosarcoma in the Lung," Chest, Vol. 103, No. 5, 1993, pp. 1531-1535. doi:10.1378/chest.103.5.1531

[3] R. Wilson, S. Glaros, R. K. Brown, et al., "Complete Radiographic Response of Primary Pulmonary AngiosaRcomas Following Gemcitabine and Taxotere," Lung Cancer, Vol. 61, No. 1, 2008, pp. 131-136. doi:10.1016/j.lungcan.2007.12.006

[4] K. Kojima, I. Okamoto, S. Ushijima, et al., "Successful Treatment of Primary Pulmonary Angiosarcoma,” Chest, Vol. 124, No. 6, 2003, pp. 2397-2400. doi:10.1378/chest.124.6.2397

[5] P. Saint-Blancard, K. Hardy, A. Bonnichon, et al., "Three-Year Survival after Treatment of a Primary Pulmonary Angiosarcoma," Revue de Pneumologie Clinique, Vol. 63, No. 1, 2007, pp. 55-58.

[6] A. P. Stout, "A Method of Teaching Postgraduate Pathology and the Importance of Correlating Research Knowledge," Perspectives in Biology and Medicine, Vol. 9, No. 3, 1966, pp. 433-435.

[7] S. A. Pandit, P. N. Fiedler and J. L. Westcott, "Primary Angiosarcoma of the Lung," Annals of Diagnostic Pathology, Vol. 9, No. 5, 2005, pp. 302-304. doi:10.1016/j.anndiagpath.2005.04.001

[8] S. L. Primack, T. E. Hartman, K. S. Lee and N. L. Muller, "Pulmonary Nodules and the CT Halo Sign," Radiology, Vol. 190, No. 2, 1994, pp. 513-515.

[9] R. G. Spragg, P. L. Wolf, P. Haghighi, et al., "Angiosarcoma of the Lung with Fatal Pulmonary Hemorrhage," American Journal of Medicine, Vol. 74, No. 6, 1983, pp. 1072-1076. doi:10.1016/0002-9343(83)90821-5

[10] C. Adem, M. C. Aubry, H. D. Tazelaar and J. L. Myers, "Metastatic Angiosarcoma Masquerading as Diffuse Pulmonary Hemorrhage: Clinicopathologic Analysis of $7 \mathrm{New}$ Patients," Archives of Pathology \& Laboratory Medicine, Vol. 125, No. 12, 2001, pp. 1562-1565. 
[11] E. A. Bacha, C. D. Wright, H. C. Grillo, et al., "Surgical Treatment of Primary Pulmonary Sarcomas," European Journal of Cardio-Thoracic Surgery, Vol. 15, No. 4, 1999, pp. 456-460. doi:10.1016/S1010-7940(99)00045-7

[12] G. A. Carillo, M. A. Carretero, J. E. Vazquez, et al., "Epithelioid Angiosarcoma of the Lung with Pleural Metastases: A Rare Cause of Haemoptysis Clinicopathological Conference," Heart Lung Circ, Vol. 19, No. 10, pp. 624-628.

[13] S. L. Segal, G. S. Lenchner, A. V. Cichelli, et al., "Angiosarcoma Presenting as Diffuse Alveolar Hemorrhage,” Chest, Vol. 94, No. 1, 1988, pp. 214-216. doi:10.1378/chest.94.1.214

[14] S. A. Yousem, "Angiosarcoma Presenting in the Lung," Archives of Pathology \& Laboratory Medicine, Vol. 110, No. 2, 1986, pp. 112-115.

[15] C. A. Holden, M. F. Spittle and E. W. Jones, "Angiosarcoma of the Face and Scalp, Prognosis and Treatment," Cancer, Vol. 59, No. 5, 1987, pp. 1046-1057. doi:10.1002/1097-0142(19870301)59:5<1046::AID-CNC R2820590533>3.0.CO;2-6

[16] R. Sasaki, T. Soejima, K. Kishi, et al., “Angiosarcoma Treated with Radiotherapy: Impact of Tumor Type and Size on Outcome," International Journal of Radiation Oncology Biology Physics, Vol. 52, No. 4, 2002, pp. 1032-1040. doi:10.1016/S0360-3016(01)02753-5

[17] M. Masuzawa, N. Mochida, T. Amano, et al., "Evaluation of Recombinant Interleukin-2 Immunotherapy for Human Hemangiosarcoma in a SCID Mice Model (WB-SCID)," Journal of Dermatological Science, Vol. 27, No. 2, 2001, pp. 88-94. doi:10.1016/S0923-1811(01)00103-7

[18] H. Ihda, Y. Tokura, M. Fushimi, et al., "Malignant Hemangioendothelioma," International Journal of Dermatology, Vol. 34, No. 11, 1995, pp. 811-816. doi:10.1111/j.1365-4362.1995.tb04406.x

[19] C. Atasoy, S. Fitoz, H. Yigit, et al., "Radiographic, CT, and MRI Findings in Primary Pulmonary Angiosarcoma," Clinical Imaging, Vol. 25, No. 5, 2001, pp. 337-340. doi:10.1016/S0899-7071(01)00302-3

[20] K. M. Leu, L. J. Ostruszka, D. Shewach, et al., “Laboratory and Clinical Evidence of Synergistic Cytotoxicity of Sequential Treatment with Gemcitabine Followed by Docetaxel in the Treatment of Sarcoma," Journal of Clinical Oncology, Vol. 22, No. 9, 2004, pp. 1706-1712. doi:10.1200/JCO.2004.08.043

[21] K. Okamoto, M. Ocker, D. Neureiter, et al., "Bcl-2-Specific Sirnas Restore Gemcitabine Sensitivity in Human Pancreatic Cancer Cells,” Journal of Cellular and Molecular Medicine, Vol. 11, No. 2, 2007, pp. 349-361. doi:10.1111/j.1582-4934.2007.00013.x

[22] L. Ricotti, A. Tesei, F. De Paola, et al., "In Vitro Schedule -Dependent Interaction between Docetaxel and Gemcitabine in Human Gastric Cancer Cell Lines,” Clinical Cancer Research, Vol. 9, No. 2, 2003, pp. 900-905. 